\title{
A Computational Study of HSV-2 with Poor Treatment Adherence
}

\author{
A. Mhlanga, C. P. Bhunu, and S. Mushayabasa \\ Department of Mathematics, University of Zimbabwe, P.O. Box MP 167, Mount Pleasant, Harare, Zimbabwe
}

Correspondence should be addressed to A. Mhlanga; ngoni72@gmail.com

Received 3 July 2015; Revised 31 August 2015; Accepted 25 October 2015

Academic Editor: Yanni Xiao

Copyright (C) 2015 A. Mhlanga et al. This is an open access article distributed under the Creative Commons Attribution License, which permits unrestricted use, distribution, and reproduction in any medium, provided the original work is properly cited.

Herpes simplex virus type 2 (HSV-2) is the most prevalent sexually transmitted disease worldwide, despite the availability of highly effective antiviral treatments. In this paper, a basic mathematical model for the spread of HSV-2 incorporating all the relevant biological details and poor treatment adherence is proposed and analysed. Equilibrium states of the model are determined and their stability has been investigated. The basic model is then extended to incorporate a time dependent intervention strategy. The aim of the control is tied to reducing the rate at which HSV-2 patients in treatment quit therapy before completion. Practically, this control can be implemented through monitoring and counselling all HSV-2 patients in treatment. The Pontryagin's maximum principle is used to characterize the optimal level of the control, and the resulting optimality system is solved numerically. Overall, the study demonstrates that though time dependent control will be effective on controlling new HSV-2 cases it may not be sustainable for certain time intervals.

\section{Introduction}

Sexually transmitted infections (STIs) are responsible for an enormous burden of morbidity and mortality in many developing countries because of their effects on reproductive and child health and their role in facilitating the transmission of HIV infection [1]. Some of the reasons why STIs incidence and prevalence rates are generally high in developing nations are inadequacies in health service provision and health care seeking [2] and delayed and poor treatment adherence [3]. Adhering to a treatment schedule and successfully completing it are crucial to the control of any global disease [4]. Poor adherence to treatment can be influenced by a couple of issues such as personal, psychosocial, economic, medical, and health service factors [4]. Prior studies suggest that rates of nonadherence to treatment generally range from 20 to $40 \%$ for acute illness regimen and 50 to $80 \%$ for preventive regimens [5]. It is worth noting that poor treatment adherence has serious adverse effects on the GDP (Gross Domestic Product) of a nation; for instance, a study in United States of America (USA) estimated the total costs associated with poor treatment adherence to be in the range $\$ 100-\$ 300$ billion each year, including both direct and indirect costs $[6,7]$.

Herpes simplex virus type 2 (HSV-2) is the most prevalent sexually transmitted disease worldwide, with a prevalence of up to $80 \%$ [8]. HSV-2 causes lifelong infection with episodic reactivation. Mounting evidence indicates that HSV2 infection increases susceptibility to human immunodeficiency virus (HIV) infection [9]; thus a positive outcome on controlling HSV-2 will reduce HIV incidence and prevalence. For decades, antiviral drugs such as valacyclovir have been used to treat or prevent frequent and painful episodes [10]. There is no effective vaccine for HSV-2 yet, but the infected people may rely on the suppressive antiviral treatment, and for them to be effective and also to decrease HSV-2 shedding, adherence would be an important behaviour to follow [8, 11]. Prior studies suggest that poor treatment adherence is more common among patients taking medication with a once-daily dosing schedule compared to three or more frequent dosing, signifying that HSV-2 patients also who need to take their medication twice or three times a day are susceptible to nonadherence [12]. Plummer et al. [13] 
conducted a qualitative study on HSV-2 treatment adherence in Tanzania and observed an 8\% (7/86) adherence for HSV-2 patients who were on acyclovir. Despite numerous research efforts that have been devoted to the study of HSV$2[9,14-18]$, the aspect of poor treatment adherence and its impact on infection and spread of HSV-2 has not yet been investigated. It is against this background that this study finds its relevance. In this paper we propose a compartmental model for the spread of HSV-2 incorporating all the essential biological details. The model incorporates the aspect of poor treatment adherence and allows optimal control methods to be used. We conduct both epidemic and endemic analysis, with a focus on the threshold dynamics characterized by the basic reproduction numbers. In addition, we explored the role of time dependent intervention strategy of controlling HSV-2 incidence and prevalence.

The paper is structured as follows. Section 2 presents the basic HSV-2 model framework. In Section 3 we conduct qualitative and comprehensive analysis of the model. In Section 4 we extend the basic model to incorporate optimal control theory. A brief discussion section concludes the paper.

\section{Model Framework}

Our objective is to formulate a deterministic model for HSV-2 that includes relevant biological details, accounts for HSV-2 treatment, and allows optimal control methods to be used. To begin, we partition the sexual active population into the following epidemiological classes or subgroups: susceptibles $(\bar{S})$, individuals infected with acute HSV-2 and also under antiviral treatment $\left(\bar{A}_{a}\right)$, individuals infected with acute HSV-2 and not under antiviral treatment after quitting before completion $\left(\bar{A}_{u}\right)$, individuals infected with latent HSV2 after undergoing successful antiviral treatment $\left(\bar{L}_{a}\right)$, and the individuals infected with latent HSV-2 after undergoing natural healing $\left(\bar{L}_{u}\right)$. Thus, the total population is given by $\bar{N}=\bar{S}+\bar{A}_{a}+\bar{A}_{u}+\bar{L}_{a}+\bar{L}_{u}$. We assume a constant size population with a birth and non-disease related death rate given by $\mu$. The recruitment of susceptibles is proportional to the population and is given by $\mu \bar{N}(t)$. Assuming homogeneous mixing of the population, the susceptible individuals acquire acute HSV-2 infection at rate $\bar{\lambda}$, given by

$$
\bar{\lambda}=\frac{\beta\left(\bar{A}_{a}(1-\eta)+\bar{A}_{u}\right)}{\bar{N}},
$$

where $\beta=\rho c$ is the effective contact rate for HSV-2 infection (contact sufficient to result in HSV-2 infection), $\rho$ is the probability of being infected by a sexual partner and $c$ is the rate at which an individual acquires sexual partners per unit time, and $\eta \in(0,1)$ is a modification factor accounting for the assumed reduced likelihood for individuals in class $\bar{A}_{a}$ to pass on the infection compared to individuals in class $\bar{A}_{u}$. This is due to the fact that individuals in treatment have reduced viral load compared to those who have failed to adhere to antiviral treatment guidelines. Upon being infected with acute HSV-2, the individuals become latent at constant rate $\kappa$.
Following an appropriate stimulus in individuals with latent HSV-2, reactivation may occur [14]. The antiviral treatment rate for the individuals with acute HSV-2 is denoted by $\psi$. Since the antiviral medication will also suppress reactivation of latent HSV-2, we assume that the reactivation rate of people with latent HSV-2 is at rate $\gamma(\psi)$, where $\gamma(\psi)$ is a decreasing function of $\psi[9]$. Thus,

$$
\gamma(\psi)=\gamma(0) \frac{\alpha}{\alpha+\psi}
$$

in which the factor $\alpha /(\alpha+\psi)$ represents reduced reactivation to class $\bar{A}_{a}$ due to antiviral treatment. In the case where there is no treatment we have that $\psi=0$; thus $\gamma\left(\psi_{0}\right)=\gamma(0)=\gamma_{0}$. A proportion $(1-\theta)$ of individuals on treatment fail to adhere to treatment at rate $\delta$, and they move to class $\bar{A}_{u}$. Since HSV2 is not fatal, we assume that individuals in different human subgroups suffer from natural death at a rate $\mu$.

HSV-2 dynamics in this study are governed by the following system of nonlinear differential equations:

$$
\begin{aligned}
& \bar{S}^{\prime}=\mu \bar{N}-\bar{\lambda} \bar{S}-\mu \bar{S}, \\
& \bar{A}_{a}^{\prime}=\bar{\lambda} \bar{S}+\gamma(\psi) \bar{L}_{a}-(\mu+\kappa+\psi+\delta(1-\theta)) \bar{A}_{a}, \\
& \bar{A}_{u}^{\prime}=\delta(1-\theta) \bar{A}_{a}+\gamma_{0} \bar{L}_{u}-(\mu+\kappa) \bar{A}_{u}, \\
& \bar{L}_{a}^{\prime}=(\kappa+\psi) \bar{A}_{a}-(\mu+\gamma(\psi)) \bar{L}_{a}, \\
& \bar{L}_{u}^{\prime}=\kappa \bar{A}_{u}-\left(\mu+\gamma_{0}\right) \bar{L}_{u} .
\end{aligned}
$$

It is helpful to rescale system (3) so that we have dimensionless variables. We let

$$
\begin{aligned}
S & =\frac{\bar{S}}{\bar{N}}, \\
L_{a} & =\frac{\bar{L}_{a}}{\bar{N}}, \\
L_{u} & =\frac{\bar{L}_{u}}{\bar{N}}, \\
A_{a} & =\frac{\bar{A}_{a}}{\bar{N}}, \\
A_{u} & =\frac{\bar{A}}{\bar{N}} .
\end{aligned}
$$

We now denote the total population with $N=S+L_{a}+L_{u}+$ $A_{a}+A_{u}$, and our new model takes the form

$$
\begin{aligned}
& S^{\prime}=\mu-\lambda S-\mu S, \\
& A_{a}^{\prime}=\lambda S+\gamma(\psi) L_{a}-(\mu+\kappa+\psi+\delta(1-\theta)) A_{a}, \\
& A_{u}^{\prime}=\delta(1-\theta) A_{a}+\gamma_{0} L_{u}-(\mu+\kappa) A_{u},
\end{aligned}
$$


TABLE 1: Model parameters and their baseline values. The time unit is in months.

\begin{tabular}{|c|c|c|c|}
\hline Definition & Symbol & Baseline values & Source \\
\hline Reactivation rate with an effect of treatment & $\gamma(\psi)$ & Varying & {$[9]$} \\
\hline Proportion of HSV-2 patients who successfully complete treatment & $\theta$ & $0.7(0-1)$ & Estimate \\
\hline Effective contact rate for HSV-2 infection & $\beta$ & 0.2 & {$[9,18]$} \\
\hline Baseline reactivation rate of latent HSV-2 & $\gamma_{0}$ & $0.3875(0.339-0.436)$ & [19] \\
\hline Rate of acute HSV-2 becoming latent & $\kappa$ & $2.3805(2.083-2.678)$ & [19] \\
\hline Treatment rate of acute HSV-2 & $\psi$ & Varied & [9] \\
\hline Average sexual lifespan & $\mu$ & $0.0004(0.003-0.005)$ & {$[18,19]$} \\
\hline Modification parameter & $\eta$ & $0.4(0-1)$ & Estimate \\
\hline Treatment quitting rate & $\delta$ & 0.3 & {$[20,21]$} \\
\hline
\end{tabular}

$$
\begin{aligned}
& L_{a}^{\prime}=(\kappa+\psi) A_{a}-(\mu+\gamma(\psi)) L_{a}, \\
& L_{u}^{\prime}=\kappa A_{u}-\left(\mu+\gamma_{0}\right) L_{u}
\end{aligned}
$$

with $\lambda=\beta\left(A_{a}(1-\eta)+A_{u}\right)$. The model parameters and their baseline values are given in Table 1 .

\section{Model Analysis and Results}

3.1. Positivity and Boundedness of Solutions. In this section we study the basic properties of the solutions of model system (5), which are essential to the proofs of stability.

Theorem 1. The equations preserve positivity of solutions.

Proof. Let $t_{1}=\sup \left\{t>0: S>0, A_{a}>0, A_{u}>0, L_{a}>\right.$ $\left.0, L_{u}>0 \in[0, t]\right\}$. Thus, $t_{1}>0$. It follows from the first equation of model system (5) that

$$
\dot{S}=\mu-\mu S \geq \mu-\lambda S-\mu S,
$$

which can be rewritten as

$$
\begin{gathered}
\frac{d}{d t}\left(S(t) \exp \left\{\mu t+\int_{0}^{t} \lambda(\tau) d \tau\right\}\right) \\
\quad \geq \mu \exp \left\{\mu t+\int_{0}^{t} \lambda(\tau) d \tau\right\} .
\end{gathered}
$$

Hence,

$$
\begin{aligned}
& S\left(t_{1}\right) \exp \left\{\mu t_{1}+\int_{0}^{t} \lambda(\tau) d \tau\right\}-S(0) \\
& \quad \geq \int_{0}^{t_{1}} \mu \exp \left\{\mu y+\int_{0}^{y} \lambda(\tau) d \tau\right\} d y,
\end{aligned}
$$

so that

$$
\begin{gathered}
S\left(t_{1}\right) \geq S(0) \exp \left\{-\mu t_{1}-\int_{0}^{t_{1}} \lambda(\tau) d \tau\right\} \\
\cdot\left(\exp \left\{-\mu t_{1}-\int_{0}^{t_{1}} \lambda(\tau) d \tau\right\}\right) \\
\cdot\left(\int_{0}^{t_{1}} \mu \exp \left\{\mu y+\int_{0}^{y} \lambda(\tau)\right\} d y\right)>0 .
\end{gathered}
$$

Similarly, it can be shown that $A_{a}>0, A_{u}>0, L_{a}>0$, and $L_{u}>0$ for all time $t>0$.

Theorem 2. All solutions of model system (5) are bounded.

Proof. Using model system (5), we have

$$
N^{\prime}=S^{\prime}+A_{a}^{\prime}+A_{u}^{\prime}+L_{a}^{\prime}+L_{u}^{\prime}=\mu-\mu N .
$$

The initial value problem $\Theta^{\prime}=\mu-\mu \Theta$, with $\Theta(0)=N(0)$, has the solution $\Theta(t)=1-c e^{-\mu t}$ and $\lim _{t \rightarrow \infty} \Theta(t)=1$. Therefore, $N(t) \leq \Theta(t)$, which implies that $\lim _{t \rightarrow \infty} \sup N(t) \leq 1$.

Therefore all feasible solutions of system (5) enter the region

$$
\begin{aligned}
\Omega & =\left\{\left(S, A_{a}, A_{u}, L_{a}, L_{u}\right) \in \mathbb{R}_{+}^{5}: S+A_{a}+A_{u}+L_{a}\right. \\
& \left.+L_{u} \leq 1\right\} .
\end{aligned}
$$

Thus, $\Omega$ is positively invariant and it is sufficient to consider solutions of model system (5) in $\Omega$. Existence, uniqueness, and continuation results for model system (5) starting in $\Omega$ remain in $\Omega$ for all $t \geq 0$. All parameters and state variables for model system (5) are assumed to be nonnegative (for biological relevance) for all $t \geq 0$ since model system monitors human population.

\subsection{Equilibrium Points and Their Stability Analysis}

3.2.1. Disease-Free Equilibrium. Model system (5) has an evident disease-free equilibrium (DFE) given by

$$
\mathscr{C}^{0}=\left(S^{0}, A_{a}^{0}, A_{u}^{0}, L_{a}^{0}, L_{u}^{0}\right)=(1,0,0,0,0)
$$

The Jacobian matrix of model system (5) evaluated at the disease-free equilibrium is given by $\Pi$ : 


$$
\Pi=\left[\begin{array}{ccccc}
-\mu & -(1-\eta) \beta & -\beta & 0 & 0 \\
0 & (1-\eta) \beta-(\delta(1-\theta)+\mu+\kappa+\psi) & \beta & \gamma(\psi) & 0 \\
0 & \delta(1-\theta) & -(\mu+\kappa) & 0 & \gamma_{0} \\
0 & \kappa+\psi & 0 & -(\mu+\gamma(\psi)) & 0 \\
0 & 0 & \kappa & 0 & -\left(\mu+\gamma_{0}\right)
\end{array}\right] .
$$

It is clear from (13) that $\lambda_{1}=-\mu$ is an eigenvalue and the other four eigenvalues are obtained from the matrix

$$
\Delta=\left[\begin{array}{cccc}
(1-\eta) \beta-(\delta(1-\theta)+\mu+\kappa+\psi) & \beta & \gamma(\psi) & 0 \\
\delta(1-\theta) & -(\mu+\kappa) & 0 & \gamma_{0} \\
\kappa+\psi & 0 & -(\mu+\gamma(\psi)) & 0 \\
0 & \kappa & 0 & -\left(\mu+\gamma_{0}\right)
\end{array}\right]
$$

The trace and determinant of (14) are, respectively, given by

$$
\begin{aligned}
\operatorname{tr}(\Delta) & =-\left(4 \mu+2 \kappa+\psi+\gamma(\psi)+\gamma_{0}+\delta(1-\theta)\right)+(1-\eta) \beta<0, \text { for }(1-\eta) \beta<(\delta(1-\theta)+\mu+\kappa+\psi), \\
\operatorname{det}(\Delta) & =\omega\left(1-\frac{\beta(\mu+\gamma(\psi))\left[\delta(1-\theta)\left(\mu+\gamma_{0}\right)+\mu(1-\eta)\left(\mu+\kappa+\gamma_{0}\right)\right]}{\mu\left(\mu+\kappa+\gamma_{0}\right)[\gamma(\psi)(\delta(1-\theta)+\mu)+\mu(\delta(1-\theta)+\mu+\kappa+\psi)]}\right)>0,
\end{aligned}
$$

where, $\Phi=\mu\left(\mu+\kappa+\gamma_{0}\right)[\gamma(\psi)(\delta(1-\theta)+\mu)+\mu(\delta(1-\theta)+\mu+\kappa+\psi)]$.

$$
R_{a c}=\frac{\beta(\mu+\gamma(\psi))\left[\delta(1-\theta)\left(\mu+\gamma_{0}\right)+\mu(1-\eta)\left(\mu+\kappa+\gamma_{0}\right)\right]}{\mu\left(\mu+\kappa+\gamma_{0}\right)[\gamma(\psi)(\delta(1-\theta)+\mu)+\mu(\delta(1-\theta)+\mu+\kappa+\psi)]} .
$$

Biologically, the reproductive number is defined as the number of secondary cases generated by a primary case when the virus is introduced in a population of wholly susceptible individuals at a demographic steady state. Based on (15) we have Theorem 3.

Theorem 3. If $R_{a c}<1$, then $\mathscr{C}^{0}$ is locally asymptotically stable.

We now investigate the global stability of the infectionfree equilibrium $\mathscr{C}^{0}$. We claim the following result.

Theorem 4. Whenever $R_{a c}<1$, it follows that the equilibrium point $\mathscr{C}^{0}$ of system (5) is globally asymptotically stable.
Proof. From the first equation of model system (5), we have that

$$
S^{\prime}=\mu-\beta\left(A_{a}(1-\eta)+A_{u}\right) S-\mu S \leq \mu-\mu S .
$$

Choosing a small enough positive number $v$, there exists $t_{1}>$ 0 such that, for all $t>t_{1}$,

$$
S \leq 1+v=S^{0}+v
$$


From model system (5) and the fact that $S \leq S^{0}+v, t>t_{1}$, we also know that

$$
\begin{aligned}
A_{a}^{\prime} \leq & \beta\left(A_{a}(1-\eta)+A_{u}\right)\left(S^{0}+v\right)+\gamma(\psi) L_{a} \\
& -(\mu+\kappa+\psi+\delta(1-\theta)) A_{a}, \\
A_{u}^{\prime}= & \delta(1-\theta) A_{a}+\gamma_{0} L_{u}-(\mu+\kappa) A_{u}, \\
L_{a}^{\prime}= & (\kappa+\psi) A_{a}-(\mu+\gamma(\psi)) L_{a}, \\
L_{u}^{\prime}= & \kappa A_{u}-\left(\mu+\gamma_{0}\right) L_{u}
\end{aligned}
$$

for all $t>t_{1}$. Consider the following auxiliary system:

$$
\begin{aligned}
A_{a}^{\prime}= & \beta\left(A_{a}^{* *}(1-\eta)+A_{u}^{* *}\right)\left(S^{0}+v\right)+\gamma(\psi) L_{a}^{* *} \\
& -(\mu+\kappa+\psi+\delta(1-\theta)) A_{a}^{* *}, \\
A_{u}^{\prime}= & \delta(1-\theta) A_{a}^{* *}+\gamma_{0} L_{u}^{* *}-(\mu+\kappa) A_{u}^{* *}, \\
L_{a}^{\prime}= & (\kappa+\psi) A_{a}^{* *}-(\mu+\gamma(\psi)) L_{a}^{* *}, \\
L_{u}^{\prime}= & \kappa A_{u}^{* *}-\left(\mu+\gamma_{0}\right) L_{u}^{* *} .
\end{aligned}
$$

Let $\Delta_{0}$ be the matrix defined as

$$
\Delta_{0}=\left[\begin{array}{cccc}
(1-\eta) \beta & \beta & 0 & 0 \\
0 & 0 & 0 & 0 \\
0 & 0 & 0 & 0 \\
0 & 0 & 0 & 0
\end{array}\right] .
$$

Setting $\Delta_{1}=\Delta+v \Delta_{0}$, it follows from Theorem 2 in van den Driessche and Watmough [22] that $R_{a c}<1$ if and only if $\rho(\Delta)<0$. Thus, there exists $v>0$ small enough such that $\rho\left(\Delta_{1}\right)<0$. By the Perron-Frobenius theorem [23], all eigenvalues of the matrix $\Delta_{1}$ have negative real parts where $\rho\left(\Delta_{1}\right)<0$. Therefore auxiliary system has $\left(A_{a}^{\prime}(t), A_{u}^{\prime}(t), L_{a}^{\prime}(t), L_{u}^{\prime}(t)\right) \rightarrow(0,0,0,0)$, as $t \rightarrow \infty$, which implies that the zero solution of model system (5) is globally asymptotically stable.

By the comparison principle of Smith and Waltman [24], we know that $\left(A_{a}(t), A_{u}(t), L_{a}(t), L_{u}(t)\right) \rightarrow(0,0,0,0)$, at $t \rightarrow \infty$. By the theory of asymptotic autonomous system of Thieme [25], it is also known that $S(t) \rightarrow S^{0}$, as $t \rightarrow \infty$. So $\mathscr{C}^{0}$ is globally attractive when $R_{a c}<1$. It follows that the disease-free equilibrium $\mathscr{C}^{0}$ of model system (5) is globally asymptotically stable when $R_{a c}<1$.

3.2.2. Endemic Equilibrium. The endemic equilibrium of model system (5) is given by

$$
\mathscr{C}^{*}=\left\{\begin{array}{l}
S=\frac{\mu}{\mu+\lambda^{*}}, \\
L_{a}=\frac{\mu(\kappa+\psi) \lambda^{*}}{\left(\mu+\lambda^{*}\right)[\gamma(\delta(1-\theta)+\mu)+\mu(\delta(1-\theta)+\kappa+\mu+\psi)]}, \\
L_{u}=\frac{\mu \kappa \delta(1-\theta)(\mu+\gamma) \lambda^{*}}{\left(\mu+\lambda^{*}\right)\left(\kappa+\mu+\gamma_{0}\right)[\gamma(\delta(1-\theta)+\mu)+\mu(\delta(1-\theta)+\kappa+\mu+\psi)]} \\
A_{a}=\frac{\mu(\mu+\gamma) \lambda^{*}}{\left(\mu+\lambda^{*}\right)[\gamma(\delta(1-\theta)+\mu)+\mu(\delta(1-\theta)+\kappa+\mu+\psi)]} \\
A_{u}=\frac{\mu \delta(1-\theta)\left(\mu+\gamma_{0}\right)(\mu+\gamma) \lambda^{*}}{\left(\mu+\lambda^{*}\right)[\gamma(\delta(1-\theta)+\mu)+\mu(\delta(1-\theta)+\kappa+\mu+\psi)]}
\end{array}\right.
$$

In terms of the force of infection $\lambda^{*}$. Substituting (22) into the equation of the force of infection $\lambda^{*}$ we have

$$
\lambda^{*} h\left(\lambda^{*}\right)=\lambda^{*}\left(\mathscr{H}_{1} \lambda^{*}+\mathscr{H}_{2}\right)=0,
$$

where $\lambda^{*}=0$ corresponds to the disease-free equilibrium and $h\left(\lambda^{*}\right)=0$ corresponds to the existence of the endemic equilibrium point, where

$$
\begin{aligned}
& \mathscr{H}_{1}=\frac{1}{\mu}, \\
& \mathscr{H}_{2}=1-R_{a c} .
\end{aligned}
$$

$\mathscr{H}_{1}$ is always positive and $\mathscr{H}_{2}$ is negative or positive depending on whether $R_{a c}$ is greater or less than unity.
Theorem 5. The endemic equilibrium $\mathscr{C}^{*}$ exists whenever $R_{a c}>1$.

Proof. By looking at the linear equation of $\mathscr{H}_{1} \lambda^{*}+\mathscr{H}_{2}=0$ we have that

$$
\lambda^{*}=-\frac{\mathscr{H}_{2}}{\mathscr{H}_{1}}=\frac{R_{a c}-1}{\mathscr{H}_{1}} .
$$

But the disease is endemic when the force of infection $\lambda^{*}>$ 0 which implies that $R_{a c}>1$. Therefore the endemic equilibrium $\mathscr{C}^{*}$ exists whenever $R_{a c}>1$.

Since the disease-free equilibrium is globally asymptotically stable, it is evident that $\mathscr{C}^{*}$ is unique, and it has been shown that our endemic equilibrium exists for $R_{a c}>1$. When $R_{a c}>1$, the disease-free equilibrium becomes unstable and it 
is natural to expect that the infectious populations will remain persistent in this case.

Theorem 6. If $R_{a c}>1$, system (5) is uniformly persistent; namely, there exists a constant $\xi>0$ such that

$$
\begin{array}{r}
\lim \inf _{t \rightarrow \infty} S(t)>\xi, \\
\lim \inf _{t \rightarrow \infty} A_{a}(t)>\xi, \\
\lim \inf _{t \rightarrow \infty} A_{u}(t)>\xi, \\
\lim \inf _{t \rightarrow \infty} L_{a}(t)>\xi, \\
\lim \inf _{t \rightarrow \infty} L_{u}(t)>\xi .
\end{array}
$$

Here, $\xi$ is independent of the initial data in $\Omega$.

Proof. When $t \rightarrow \infty$, from system (5), we obtain the following limiting system:

$$
\begin{aligned}
S^{\prime}= & \mu-\beta S\left(A_{a}(1-\eta)+A_{u}\right)-\mu S, \\
A_{a}^{\prime}= & \beta S\left(A_{a}(1-\eta)+A_{u}\right)+\gamma(\psi) \\
& -(\mu+\kappa+\psi+\delta(1-\theta)) A_{a}, \\
A_{u}^{\prime}= & \delta(1-\theta) A_{a}+\gamma_{0} L_{u}-(\mu+\kappa) A_{u}, \\
L_{a}^{\prime}= & (\kappa+\psi) A_{a}-(\mu+\gamma(\psi)) L_{a}, \\
L_{u}^{\prime}= & \kappa A_{u}-\left(\mu+\gamma_{0}\right) L_{u} .
\end{aligned}
$$

We will also denote the disease-free equilibrium for model system (27) as $\mathscr{C}^{0}$. We set

$$
\begin{aligned}
X & =\left\{\left(S, A_{a}, A_{u}, L_{a}, L_{u}\right) \in S \geq 0, A_{a} \geq 0, A_{u} \geq 0, L_{a}\right. \\
& \left.\geq 0, L_{u} \geq 0\right\} \\
X_{0} & =\left\{\left(S, A_{a}, A_{u}, L_{a}, L_{u}\right) \in S \geq 0, A_{a}>0, A_{u}>0, L_{a}\right. \\
& \left.>0, L_{u}>0\right\} \\
Y & =X \backslash X_{0} .
\end{aligned}
$$

Now, we have to show that model system (27) is uniformly persistent with respect to $\left(X_{0}, Y\right)$.

Firstly, by the form of model system (27), it can be easily shown that both $X$ and $X_{0}$ are positively invariant. Clearly, $Y$ is relatively closed in $X$ and model system (27) is point dissipative. Consider the following set using solutions $\left(S(t), A_{a}(t), A_{u}(t), L_{a}(t), L_{u}(t)\right)$ of model system (27):

$$
\begin{aligned}
& M_{Y}=\left\{\left(S(0), A_{a}(0), A_{u}(0), L_{a}(0), L_{u}(0)\right):\right. \\
& \left.\quad\left(S(t), A_{a}(t), A_{u}(t), L_{a}(t), L_{u}(t)\right) \in Y, \forall t \geq 0\right\} .
\end{aligned}
$$

We now show that

$$
\begin{aligned}
& M_{Y} \\
& \quad=\left\{\left(S(0), A_{a}(0), A_{u}(0), L_{a}(0), L_{u}(0)\right): S \geq 0\right\} .
\end{aligned}
$$

Assume that $\left(S(0), A_{a}(0), A_{u}(0), L_{a}(0), L_{u}(0)\right) \in M_{Y}$. It suffices to show that $A_{a}(t)=A_{u}(t)=L_{a}(t)=L_{u}(t)=0$. Suppose the assumption is not true; then there exists $t_{0} \geq 0$ such that

$$
\begin{aligned}
& \left(A_{a}>0, A_{u}=0, L_{a}=0, L_{u}=0\right) \\
& \text { or }\left(A_{a}>0, A_{u}>0, L_{a}=0, L_{u}=0\right) \\
& \text { or }\left(A_{a}>0, A_{u}>0, L_{a}>0, L_{u}=0\right) \\
& \text { or }\left(A_{a}>0, A_{u}=0, L_{a}>0, L_{u}>0\right) \\
& \text { or }\left(A_{a}>0, A_{u}=0, L_{a}=0, L_{u}>0\right) \\
& \text { or }\left(A_{a}>0, A_{u}>0, L_{a}=0, L_{u}>0\right) \\
& \text { or }\left(A_{a}>0, A_{u}=0, L_{a}>0, L_{u}=0\right) \\
& \text { or }\left(A_{a}=0, A_{u}>0, L_{a}=0, L_{u}=0\right) \\
& \text { or }\left(A_{a}=0, A_{u}>0, L_{a}>0, L_{u}=0\right) \\
& \text { or }\left(A_{a}=0, A_{u}>0, L_{a}=0, L_{u}>0\right) \\
& \text { or }\left(A_{a}=0, A_{u}>0, L_{a}>0, L_{u}=0\right) \\
& \text { or }\left(A_{a}=0, A_{u}=0, L_{a}>0, L_{u}=0\right) \\
& \text { or }\left(A_{a}=0, A_{u}=0, L_{a}>0, L_{u}>0\right) \\
& \text { or }\left(A_{a}=0, A_{u}=0, L_{a}=0, L_{u}>0\right) .
\end{aligned}
$$

For $\left(A_{a}>0, A_{u}=0, L_{a}=0, L_{u}=0\right)$, we have

$$
\begin{aligned}
& A_{u}^{\prime}\left(t_{0}\right)=\delta(1-\theta) A_{a}, \\
& L_{a}^{\prime}\left(t_{0}\right)=(\kappa+\psi) A_{a} .
\end{aligned}
$$

It follows that there is $\epsilon_{0}>0$ such that $A_{u}>0$ and $L_{a}>0$ for $t_{0}<t<t_{0}+\epsilon_{0}$. Clearly, we can restrict $\epsilon_{0}>0$ small enough such that $A_{a}>0$ for $t_{0}<t<$ $t_{0}+\epsilon_{0}$. This means that $\left(S(t), A_{a}(t), A_{u}(t), L_{a}(t), L_{u}(t)\right) \notin Y$ for $t_{0}<t<t_{0}+\epsilon_{0}$, which contradicts the assumption that $\left(S(0), A_{a}(0), A_{u}(0), L_{a}(0), L_{u}(0)\right) \in M_{Y}$. For the other cases, we can also show these contradict the assumption that $\left(S(0), A_{a}(0), A_{u}(0), L_{a}(0), L_{u}(0)\right) \in M_{Y}$, respectively. Thus, (27) holds.

It is worth noting that $\mathscr{C}^{0}$ is globally asymptotically stable in the interior of $M_{Y}$. Moreover, $\mathscr{C}^{0}$ is an isolated invariant set in $X$, every orbit in $M_{Y}$ converges to $\mathscr{C}^{0}$, and $\mathscr{C}^{0}$ is acyclic in $M_{Y}$. By Theorem 4.6 [25], we only need to show that $W^{S}\left(\mathscr{C}^{0}\right) \cap X_{0}=\emptyset$ if $R_{a c}>1$.

In the following, we now prove that $W^{S}\left(\mathscr{C}^{0}\right) \cap X_{0}=\emptyset$. Assume that $W^{S}\left(\mathscr{C}^{0}\right) \cap X_{0} \neq \emptyset$. Then there exists a positive solution $\left(S^{\star}(t), A_{a}^{\star}(t), A_{u}^{\star}(t), L_{a}^{\star}(t), L_{u}^{\star}(t)\right)$ with $\left(S^{\star}(0), A_{a}^{\star}(0), A_{u}^{\star}(0), L_{a}^{\star}(0), L_{u}^{\star}(0)\right) \in X_{0}$, such that $\left(S^{\star}(t), A_{a}^{\star}(t), A_{u}^{\star}(t), L_{a}^{\star}(t), L_{u}^{\star}(t)\right) \rightarrow \mathscr{C}^{0}$ as $t \rightarrow \infty$. Since $R_{a c}>1$, we can choose $v>0$ small enough such that

$$
R_{a c}-v R_{a c}>1
$$


Thus, when $t$ is sufficiently large, we have

$$
\begin{aligned}
& S^{0}-\nu \leq S^{\star}(t) \leq S^{0}+v, \\
& 0 \leqslant A_{a}^{\star}(t) \leqslant v, \\
& 0 \leqslant A_{u}^{\star}(t) \leqslant v, \\
& 0 \leqslant L_{a}^{\star}(t) \leqslant v, \\
& 0 \leqslant L_{u}^{\star}(t) \leqslant v, \\
& A_{a}^{\prime} \geqslant \beta S\left(A_{a}(1-\eta)+A_{u}\right)+\gamma(\psi) \\
& \quad-(\mu+\kappa+\psi+\delta(1-\theta)) A_{a}, \\
& A_{u}^{\prime} \geqslant \delta(1-\theta) A_{a}+\gamma_{0} L_{u}-(\mu+\kappa) A_{u}, \\
& L_{a}^{\prime} \geqslant(\kappa+\psi) A_{a}-(\mu+\gamma(\psi)) L_{a}, \\
& L_{u}^{\prime} \geqslant \kappa A_{u}-\left(\mu+\gamma_{0}\right) L_{u} .
\end{aligned}
$$

By the comparison principle, it is easy to see that $A_{a}^{\star}(t) \rightarrow$ $\infty, A_{u}^{\star}(t) \rightarrow \infty, L_{a}^{\star}(t) \rightarrow \infty$, and $L_{u}^{\star}(t) \rightarrow \infty$, contradicting $A_{a}^{\star} \rightarrow 0, A_{u}^{\star} \rightarrow 0, L_{a}^{\star} \rightarrow 0$, and $L_{u}^{\star} \rightarrow 0$ as $t \rightarrow \infty$. This proves $W^{S}\left(\mathscr{C}^{0}\right) \cap X_{0}=\emptyset$.

Since $W^{S}\left(\mathscr{C}^{0}\right) \cap X_{0}=\emptyset, \bigcup_{x \in M_{Y}} \omega(x)=\left\{\mathscr{C}^{0}\right\}, \mathscr{C}^{0}$ is isolated invariant set in $X$, and $\mathscr{C}^{0}$ is acyclic in $M_{Y}$, by Theorem 4.6 [25] we are able to conclude that model system (27) is uniformly persistent with respect to $\left(X_{0}, Y\right)$. Then, model system (5) is uniformly persistent.

Theorem 7. If $R_{a c}>1$, the endemic equilibrium point is globally asymptotically stable.

Proof. To study the global asymptotic stability of the endemic equilibrium, following $[26,27]$ we use a Lyapunov function $V$ as follows:

$$
\begin{aligned}
V= & \left(S-S^{*}-S^{*} \ln \frac{S}{S^{*}}\right) \\
& +V_{1}\left(A_{a}-A_{a}^{*}-A_{a}^{*} \ln \frac{A_{a}}{A_{a}^{*}}\right) \\
& +V_{2}\left(A_{u}-A_{u}^{*}-A_{u}^{*} \ln \frac{A_{u}}{A_{u}^{*}}\right) \\
& +V_{3}\left(L_{a}-L_{a}^{*}-L_{a}^{*} \ln \frac{L_{a}}{L_{a}^{*}}\right) \\
& +V_{4}\left(L_{u}-L_{u}^{*}-L_{u}^{*} \ln \frac{L_{u}}{L_{u}^{*}}\right),
\end{aligned}
$$

where $V_{1}, V_{2}, V_{3}, V_{4}>0$ are constants to be determined later. Applying the replacements

$$
\begin{aligned}
& \frac{S}{S^{*}}=m, \\
& \frac{A_{a}}{A_{a}^{*}}=n, \\
& \frac{A_{u}}{A_{u}^{*}}=x, \\
& \frac{L_{a}}{L_{a}^{*}}=y, \\
& \frac{L_{u}}{L_{u}^{*}}=z,
\end{aligned}
$$

the derivative of $V$ is given by

$$
\begin{aligned}
\dot{V} & =\left(1-\frac{S^{*}}{S}\right) \frac{d S}{d t}+V_{1}\left(1-\frac{A_{a}^{*}}{A_{a}}\right) \frac{d A_{a}}{d t}+V_{2}(1 \\
& \left.-\frac{A_{u}^{*}}{A_{u}}\right) \frac{d A_{u}}{d t}+V_{3}\left(1-\frac{L_{a}^{*}}{L_{a}}\right) \frac{d L_{a}}{d t}+V_{4}(1 \\
& \left.-\frac{L_{u}^{*}}{L_{u}}\right) \frac{d L_{u}}{d t} \\
= & \left(1-\frac{1}{m}\right)\left[\mu-\beta A_{a}(1-\eta) S-\beta A_{u} S-\mu S\right] \\
& +V_{1}\left(1-\frac{1}{n}\right)\left[\beta A_{a}(1-\eta) S+\beta A_{u} S+\gamma(\psi) L_{a}\right. \\
& \left.-(\mu+\kappa+\psi+\delta(1-\theta)) A_{a}\right]+V_{2}\left(1-\frac{1}{x}\right) \\
& \cdot\left[\delta(1-\theta) A_{a}+\gamma_{0} L_{u}\right. \\
& \left.-(\mu+\kappa) A_{u}\right]+V_{3}\left(1-\frac{1}{y}\right)\left[(\kappa+\psi) A_{a}\right. \\
& \left.-(\mu+\gamma(\psi)) L_{a}\right]+V_{4}\left(1-\frac{1}{z}\right)\left[\kappa A_{u}\right. \\
& -\left(\mu L_{u}\right] .
\end{aligned}
$$

At the endemic equilibrium point, model system (5) has the following identities:

$$
\begin{aligned}
& \mu=\beta A_{a}^{*}(1-\eta) S^{*}+\beta A_{u}^{*} S^{*}+\mu S^{*}, \\
& (\mu+\kappa+\psi+\delta(1-\theta)) A_{a}^{*} \\
& \quad=\beta A_{a}^{*}(1-\eta) S^{*}+\beta A_{u}^{*} S^{*}+\gamma L_{a}^{*}, \\
& (\mu+\kappa) A_{u}^{*}=\delta(1-\theta) A_{a}^{*}+\gamma_{0} L_{u}^{*}, \\
& (\mu+\gamma(\psi)) L_{a}^{*}=(\kappa+\psi) A_{a}^{*}, \\
& \left(\mu+\gamma_{0}\right) L_{u}^{*}=\kappa A_{u}^{*} .
\end{aligned}
$$


Thus,

$$
\begin{aligned}
& \dot{V}=\left(1-\frac{1}{m}\right)\left[\beta A_{a}^{*}(1-\eta) S^{*}+\beta A_{u}^{*} S^{*}+\mu S^{*}\right. \\
& \left.-\beta A_{a}^{*}(1-\eta) S^{*} m n-\beta A_{u}^{*} S^{*} m x-\mu S^{*} m\right] \\
& +V_{1}\left(1-\frac{1}{n}\right)\left[\beta A_{a}^{*}(1-\eta) S^{*} m n+\beta A_{u}^{*} S^{*} m x\right. \\
& \left.+\gamma L_{a}^{*} y-\beta A_{a}^{*}(1-\eta) S^{*} n-\beta A_{u}^{*} S^{*} n-\gamma L_{a}^{*} n\right] \\
& +V_{2}\left(1-\frac{1}{x}\right)\left[\delta(1-\theta) A_{a}^{*} n+\gamma_{0} L_{u}^{*} z\right. \\
& \left.-\delta(1-\theta) A_{a}^{*} x-\gamma_{0} L_{u}^{*} x\right]+V_{3}\left(1-\frac{1}{y}\right) \\
& \cdot\left[(\kappa+\psi) A_{a}^{*} n-(\kappa+\psi) A_{a}^{*} y\right] \\
& +V_{4}\left(1-\frac{1}{z}\right)\left[\kappa A_{u}^{*} x-\kappa A_{u}^{*} z\right] \\
& =\beta A_{a}^{*}(1-\eta) S^{*}+\beta A_{u}^{*} S^{*}+\mu S^{*}-\beta A_{a}^{*}(1-\eta) \\
& \cdot S^{*} m n-\beta A_{u}^{*} S^{*} m x-\mu S^{*} m-\beta A_{a}^{*}(1-\eta) S^{*} \frac{1}{m} \\
& -\beta A_{u}^{*} S^{*} \frac{1}{m}-\mu S^{*} \frac{1}{m}+\beta A_{a}^{*}(1-\eta) S^{*} n \\
& +\beta A_{u}^{*} S^{*} x+\mu S^{*}+V_{1}\left(\beta A_{a}^{*}(1-\eta) S^{*} m n\right. \\
& +\beta A_{u}^{*} S^{*} m x+\gamma L_{a}^{*} y-\beta A_{a}^{*}(1-\eta) S^{*} n-\beta A_{u}^{*} S^{*} n \\
& -\gamma L_{a}^{*} n-\gamma L_{a}^{*} \frac{y}{n}-\beta A_{a}^{*}(1-\eta) S^{*} m-\beta A_{a}^{*} S^{*} \frac{m x}{n} \\
& \left.+\beta A_{a}^{*}(1-\eta) S^{*}+\beta A_{a}^{*} S^{*}+\gamma L_{a}^{*}\right) \\
& +V_{2}\left(\delta(1-\theta) A_{a}^{*} n+\gamma_{0} L_{u}^{*} z-\delta(1-\theta) A_{a}^{*} x\right. \\
& -\gamma_{0} L_{u}^{*} x-\delta(1-\theta) A_{a}^{*} \frac{n}{x}-\gamma_{0} L_{u}^{*} \frac{z}{x}+\delta(1-\theta) A_{a}^{*} \\
& \left.+\gamma_{0} L_{u}^{*}\right)+V_{3}\left((\kappa+\psi) A_{a}^{*} n-(\kappa+\psi) A_{a}^{*} y\right. \\
& \left.-(\kappa+\psi) A_{a}^{*} \frac{n}{y}+(\kappa+\psi) A_{a}^{*}\right)+V_{4}\left(\kappa A_{u}^{*} x\right. \\
& \left.-\kappa A_{u}^{*} z-\kappa A_{u}^{*} \frac{x}{z}+\kappa A_{u}^{*}\right) .
\end{aligned}
$$

We define the set $\mathscr{G}$ of all the above terms as

$$
\mathscr{G}=\left\{m, n, x, y, z, \frac{1}{m}, \frac{y}{n}, \frac{n}{y}, \frac{x}{z}, \frac{z}{x}, \frac{n}{x}, \frac{m x}{n}\right\} .
$$

Then we create some subsets of $\mathscr{G}$, where the product of each set equals one (like $(y / n) \times(n / y)=1)$. Consider the following:

$$
\begin{gathered}
\left\{m, \frac{1}{m}\right\}, \\
\left\{\frac{y}{n}, \frac{n}{y}\right\}, \\
\left\{\frac{x}{z}, \frac{z}{x}\right\}, \\
\left\{\frac{1}{m}, \frac{n}{x}, \frac{m x}{n}\right\} .
\end{gathered}
$$

Then, we let the coefficients of the terms that are not members of our subsets in (41) for the function $\dot{V}$ be zero, that is, the coefficients of the terms $n, x, y, z, m n, m x$. Thus, we have

$$
\begin{aligned}
& \left(V_{1}-1\right) \beta A_{u}^{*} S^{*}=0, \\
& \left(V_{1}-1\right) \beta A_{a}^{*}(1-\eta) S^{*}=0, \\
& \gamma L_{a}^{*} V_{1}-(\kappa+\psi) A_{a}^{*} V_{3}=0, \\
& \gamma_{0} L_{u}^{*} V_{2}-\kappa A_{u}^{*} V_{4}=0 \\
& \beta A_{u}^{*} S^{*}-\delta(1-\theta) A_{a}^{*} V_{2}-\gamma_{0} L_{u}^{*} V_{2}+\kappa A_{u}^{*} V_{4}=0, \\
& \beta A_{a}^{*}(1-\eta) S^{*}-\beta A_{a}^{*}(1-\eta) S^{*} V_{1}-\beta A_{u}^{*} S^{*} V_{1} \\
& \quad-\gamma L_{a}^{*} V_{1}+\delta(1-\theta) A_{a}^{*} V_{2}+(\kappa+\psi) A_{a}^{*} V_{3}=0 .
\end{aligned}
$$

From (42), it follows that

$$
\begin{aligned}
& V_{1}=1, \\
& V_{2}=\frac{\beta A_{u}^{*} S^{*}}{\delta(1-\theta) A_{a}^{*}}, \\
& V_{3}=\frac{\gamma L_{a}^{*}}{(\kappa+\psi) A_{a}^{*}}, \\
& V_{4}=\frac{\gamma_{0} L_{u}^{*} \beta A_{u}^{*} S^{*}}{\kappa A_{u}^{*} \delta(1-\theta) A_{a}^{*}} .
\end{aligned}
$$

From (43), the derivative of $V$ is given by

$$
\begin{aligned}
\dot{V} & =\beta A_{a}^{*}(1-\eta) S^{*}+2 \beta A_{u}^{*} S^{*}+\mu S^{*}-\mu S^{*} m \\
& -\beta A_{a}^{*}(1-\eta) S^{*} \frac{1}{m}-\beta A_{u}^{*} S^{*} \frac{1}{m}-\mu S^{*} \frac{1}{m}+\mu S^{*} \\
& +V_{1}\left(-\beta A_{u}^{*} S^{*} \frac{m x}{n}-\gamma L_{a}^{*} \frac{y}{n}+\beta A_{a}^{*}(1-\eta) S^{*}\right. \\
& \left.+\beta A_{u}^{*} S+\gamma L_{a}^{*}\right)+V_{2}\left(\gamma_{0} L_{u}^{*}+\delta(1-\theta) A_{a}^{*}\right. \\
& \left.-\gamma_{0} L_{u}^{*} \frac{z}{x}-\delta(1-\theta) A_{a}^{*} \frac{n}{x}\right)+V_{3}\left((\kappa+\psi) A_{a}^{*}\right. \\
& \left.-(\kappa+\psi) A_{a}^{*}\right)+V_{4}\left(\kappa A_{u}^{*}-\kappa A_{u}^{*} \frac{x}{z}\right) .
\end{aligned}
$$


After some tedious algebraic manipulations, we have

$$
\begin{aligned}
\dot{V}= & \left(\beta A_{a}^{*}(1-\eta) S^{*}+\mu S^{*}\right)\left(2-m-\frac{1}{m}\right) \\
& +\gamma_{0} L_{a}^{*}\left(2-\frac{n}{y}-\frac{y}{n}\right) \\
& +\frac{\gamma_{0} L_{u} \beta A_{u}^{*} S^{*}}{\delta(1-\theta) A_{a}^{*}}\left(2-\frac{z}{x}-\frac{x}{z}\right) \\
& +\beta A_{u}^{*} S^{*}\left(3-\frac{1}{m}-\frac{n}{x}-\frac{m x}{n}\right) .
\end{aligned}
$$

By the property that the arithmetic mean is greater than or equal to the geometric mean, then $2-m-1 / m \leq 0$ for $m>0$ and $2-m-1 / m=0$ if and only if $m=1 ; 3-1 / m-n / x-m x / n \leq$ 0 for $m>0, n>0$, and $x>0$ and $3-1 / m-n / x-m x / n=0$ if and only if $m=n=x=1 ; 2-x / z-z / x \leq 0$ for $x>0, z>0$, and $2-x / z-z / x=0$ if and only if $z=1$ and $x=1$, and lastly $2-y / n-n / y \leq 0$ for $n>0, y>0$, and $2-y / n-n / y=0$ if and only if $n=1$ and $y=1$.

In conclusion, the limit cycle $\dot{V}=0$ if and only if $m=n=x=y=z=1$. The maximum invariant set of system (5) on the set $\{(m, n, x, y, z): \dot{V}=0\}$ is the singleton $(1,1,1,1,1)$. Thus, for model system (5), the endemic equilibrium is globally asymptotically stable if $R_{a c}>$ 1 by La Salle invariance principle.

$$
\begin{aligned}
R_{a c}= & \frac{\beta(\mu+\gamma(\psi))\left[\delta(1-\theta)\left(\mu+\gamma_{0}\right)+\mu(1-\eta)\left(\mu+\kappa+\gamma_{0}\right)\right]}{\mu\left(\mu+\kappa+\gamma_{0}\right)[\gamma(\psi)(\delta(1-\theta)+\mu)+\mu(\delta(1-\theta)+\mu+\kappa+\psi)]}=R_{a c}^{1}+R_{a c}^{2} \\
= & \frac{\beta(\mu+\gamma(\psi))\left(\mu+\gamma_{0}\right) \delta(1-\theta)}{\mu\left(\mu+\kappa+\gamma_{0}\right)[\gamma(\psi)(\delta(1-\theta)+\mu)+\mu(\delta(1-\theta)+\mu+\kappa+\psi)]} \\
& +\frac{\beta(1-\eta)(\mu+\gamma(\psi))}{[\gamma(\psi)(\delta(1-\theta)+\mu)+\mu(\delta(1-\theta)+\mu+\kappa+\psi)]} .
\end{aligned}
$$

3.2.3. Analysis of the Reproduction Number. In this section we theoretically assess the effects of adherence on curtailing the spread of HSV-2 and also consider some different possibilities for our reproduction numbers.

Case 1 (there is no treatment in the community). We set $\psi=$ $\eta=0$ and $\theta=1$. Then the spectral radius is given by

$$
R_{0}=\frac{\beta\left(\mu+\gamma_{0}\right)}{\mu\left(\mu+\kappa+\gamma_{0}\right)} .
$$

Biologically, $R_{0}$ represents the average number of secondary HSV-2 cases produced by a single HSV-2 infected individual during his/her infectious period in the absence of treatment and any other HSV-2 intervention strategies.

Case 2 (everyone is adhering to the HSV-2 antiviral treatment in the community). We set $\theta=1$. Then the spectral radius is given by

$$
R_{a}=\frac{\beta(\mu+\gamma(\psi))}{\mu(\gamma(\psi)+\kappa+\psi+\mu)} .
$$

Biologically, $R_{a}$ represents the average number of secondary HSV-2 cases produced by a single HSV-2 infected individual during his/her infectious period in the presence of HSV-2 treatment where everyone is adhering to the HSV-2 antiviral treatment.

Case 3 (the general case). This is the general case which has been defined in (16) above, which is given by 
TABLE 2: Sensitivity indices of model parameters to $R_{a c}$.

\begin{tabular}{lclc}
\hline & Symbol & Definition & Sensitivity index \\
\hline 1 & $\gamma(\psi)$ & Reactivation rate of latent HSV-2 with an effect of treatment & 0.327651 \\
2 & $\gamma_{0}$ & Baseline reactivation rate of latent HSV-2 & 0.691435 \\
3 & $\beta$ & Effective contact rate for HSV-2 infection & 1 \\
4 & $\kappa$ & Rate of acute HSV-2 becoming latent & -0.976583 \\
5 & $\psi$ & Treatment rate of acute HSV-2 & -0.0538677 \\
6 & $\mu$ & Average sexual lifespan & -1.01589 \\
7 & $\theta$ & Proportion of adherence & -0.51079 \\
8 & $\delta$ & Treatment quitting rate & 0.170263 \\
9 & $\eta$ & Modification parameter & -0.130296 \\
\hline
\end{tabular}

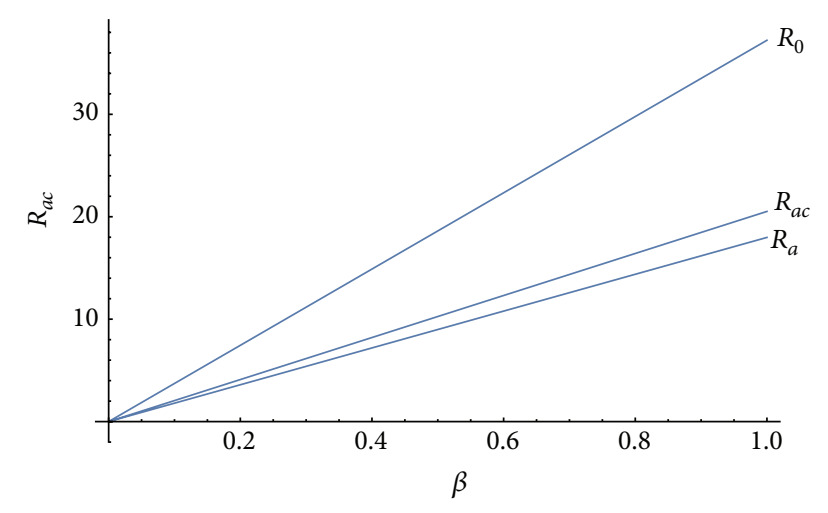

FIGURE 1: Effect of varying the effective contact rate $\beta$ on the reproduction numbers.

resulting from the evaluation to other model parameters are shown in Table 2. The parameters are arranged from the most sensitive one to the least sensitive one. The sensitivity index with respect to $\theta$, for example, is

$$
\Upsilon_{\theta}^{R_{a c}}=\frac{\partial R_{a c}}{\partial \theta} \times \frac{\theta}{R_{a c}}=-0.51079 .
$$

Since $\Upsilon_{\theta}^{R_{a c}}=-0.51079$, increasing (or decreasing) the proportion of individuals who are adhering to HSV-2 antiviral treatment by $51 \%$ increased (or decreased) the reproduction number $\left(R_{a c}\right)$ by $51 \%$. Thus, increasing the number of individuals adhering to HSV-2 antiviral treatment would be very crucial in curtailing the spread of HSV-2.

Similarly, increasing (or decreasing) the treatment rate of the acute HSV-2 infected individuals by $5 \%$ would also trigger an increase (or decrease) in $R_{a c}$ by $5 \%$. It is worth noting that an increase in treatment rate of the acute HSV2 individuals automatically reduces the reactivation rate of latent HSV-2 individuals who came into the latent class through treatment. As illustrated in Table 2, reactivation rate of the latent HSV-2 individuals who adhered to treatment has an effect of reducing the reproduction number $\left(R_{a c}\right)$ by $33 \%$ if it has been increased or decreased by $33 \%$. Thus, it would be very crucial to increase treatment rate of the acute HSV-2 individuals. Methods such as the use of prepackaged blister cards [30], the use of follow-up telephone calls [31], and sharing of simple instructions about antimicrobial/antiviral therapy which include information about adverse reactions $[32,33]$ can be employed so as to improve and encourage adherence within the community.

Figure 2(a) depicts the combined effect of the effective contact rate $\beta$ and the proportion of individuals who are adhering to the HSV- 2 antiviral treatment $\theta$ on the reproduction number $R_{a c}$. These contour plots show a marked decrease in $R_{a c}$ with increasing adherence and decreasing effective contact rate. Significantly high adherence and low effective contact rate are needed to reduce the prevalence of HSV-2 (achieving $R_{a c}<1$ ). Figure 2(b) depicts the combined effect of proportion of individuals who are adhering to the HSV-2 antiviral treatment $\theta$ and the treatment rate of acute HSV-2 on the reproduction number $R_{a c}$. Figure 2(b) shows that the reproduction number can be significantly reduced for high rates of adherence and high rates of treatment.

Figure 3 depicts that if more people are adhering to HSV-2 antiviral treatment, then the disease can be curtailed. Conversely, low value of people adhering to HSV-2 antiviral treatment implies that HSV-2 will prevail.

\section{Optimal Control Problem}

We introduce into model system (5) a time dependent control effort on counselling and monitoring $(u)$, as a control to curtail the spread of HSV-2. HSV-2 model system (5) becomes

$$
\begin{aligned}
S^{\prime} & =\mu-\lambda S-\mu S, \\
A_{a}^{\prime} & =\lambda S+\gamma(\psi)-(\mu+\kappa+\psi) A_{a}-\delta(1-u) A_{a}, \\
A_{u}^{\prime} & =\delta(1-u) A_{a}+\gamma_{0} L_{u}-(\mu+\kappa) A_{u}, \\
L_{a}^{\prime} & =(\kappa+\psi) A_{a}-(\mu+\gamma(\psi)) L_{a}, \\
L_{u}^{\prime} & =\kappa A_{u}-\left(\mu+\gamma_{0}\right) L_{u} .
\end{aligned}
$$

For this, we consider the objective functional

$$
J(u)=\min _{u} \int_{0}^{t_{f}}\left(z A_{u}+\vartheta u^{2}\right) d t
$$




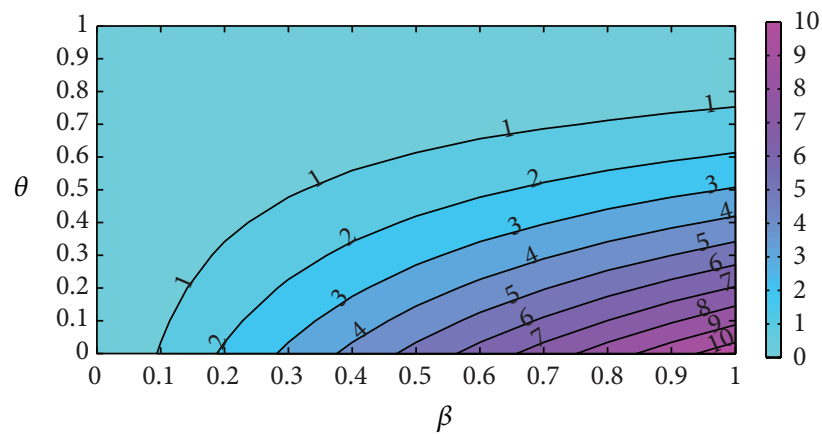

(a)

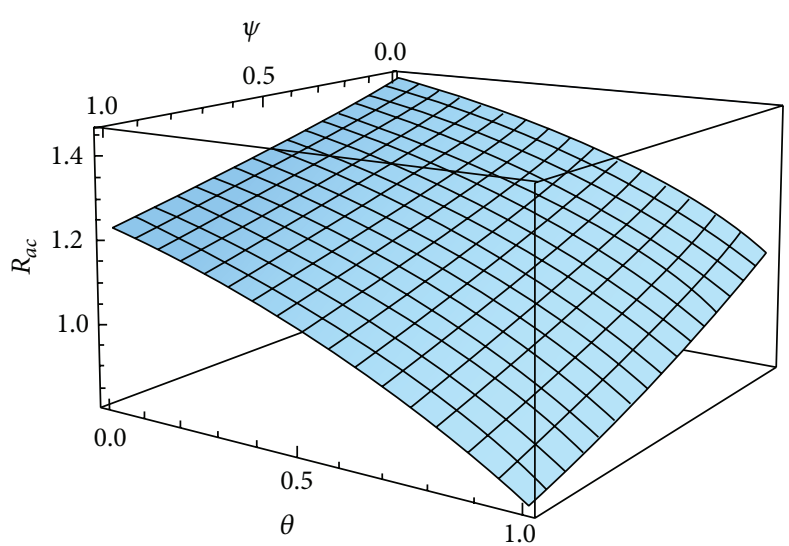

(b)

FIGURE 2: Simulations of model system (5) (a) showing the contour plots of $R_{a c}$ as a function of the effective contact rate for HSV-2 infection $(\beta)$ and the proportion of individuals who are adhering to the HSV-2 antiviral treatment $(\theta)$; (b) showing the effects of varying the treatment rate of acute HSV-2 and the proportion of individuals who are adhering to the HSV-2 antiviral treatment $(\theta)$.

where $t_{f}$ is the final time and $z$ is a positive weight constant to balance the factor. The term $\vartheta u^{2}$ is the cost of the control effort on counselling and monitoring of HSV-2 individuals under treatment. We choose a quadratic cost on the control; this is similar to that in other literature on epidemic controls $[34,35]$. With the given objective function $J(u)$, our goal is to minimize the number of HSV-2 individuals who would have failed to adhere to HSV-2 antiviral treatment $A_{u}$, while minimizing the cost of control $u^{*}$ such that

$$
J\left(u^{*}\right)=\min \{J(u) \mid u \in \mathscr{U}\}
$$

where $\mathcal{u}=\{u$ is such that $u$ is measurable with $0 \leq u \leq$ 1 for $\left.t \in\left[0, t_{f}\right]\right\}$ is the control set. The necessary conditions that an optimal control must satisfy come from Pontryagin's maximum principle [36]. This principle converts (50)-(51) into a problem of minimizing pointwise a Hamiltonian $H$, with respect to $u$ :

$$
\begin{aligned}
H & =z A_{u}+9 u^{2}+\lambda_{1}[\mu-\lambda S-\mu S]+\lambda_{2}[\lambda S \\
& \left.+\gamma(\psi) L_{a}-(\mu+\kappa+\psi) A_{a}-\delta(1-u) A_{a}\right] \\
& +\lambda_{3}\left[\delta\left(1-u_{1}\right) A_{a}+\gamma_{0} L_{u}-(\mu+\kappa) A_{u}\right] \\
& +\lambda_{4}\left[(\kappa+\psi) A_{a}-(\mu+\gamma(\psi)) L_{a}\right]+\lambda_{5}\left[\kappa A_{u}\right. \\
& \left.-\left(\mu+\gamma_{0}\right)\right],
\end{aligned}
$$

where the $\lambda_{1}, \lambda_{2}, \lambda_{3}, \lambda_{4}$, and $\lambda_{5}$ are the adjoint variables or shadow price. By applying Pontryagin's maximum principle [36] and the existence result for the optimal control from [37] we obtain the following.

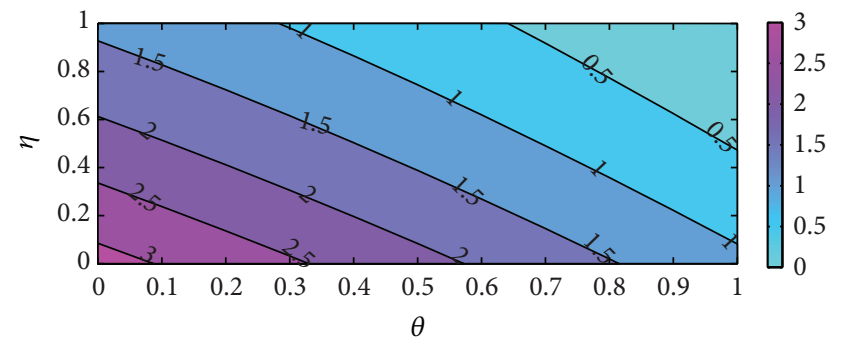

FIgURE 3: Simulations of model system (5) showing the contour plots of $R_{a c}$ as a function of the modification parameter $(\eta)$ and the proportion of individuals who are adhering to the HSV-2 antiviral treatment $(\theta)$.

Theorem 8. For the optimal control $u^{*}$ that minimizes $J(u)$ over $\mathcal{U}$, there exist adjoint variables $\lambda_{1}, \lambda_{2}, \lambda_{3}, \lambda_{4}$, and $\lambda_{5}$ satisfying

$$
\begin{aligned}
\lambda_{1}^{\prime}(t)= & \beta\left(A_{a}(1-\eta)+A_{u}\right)\left[\lambda_{1}-\lambda_{2}\right]+\mu \lambda_{1}, \\
\lambda_{2}^{\prime}(t)= & \beta(1-\eta) S\left[\lambda_{1}-\lambda_{2}\right]+(\kappa+\psi)\left[\lambda_{2}-\lambda_{4}\right] \\
& +\delta(1-u)\left[\lambda_{2}-\lambda_{3}\right]+\mu \lambda_{2}, \\
\lambda_{3}^{\prime}(t)= & -z+\beta S\left[\lambda_{1}-\lambda_{2}\right]+\kappa\left[\lambda_{3}-\lambda_{5}\right]+\mu \lambda_{3}, \\
\lambda_{4}^{\prime}(t)= & \gamma(\psi)\left[\lambda_{4}-\lambda_{2}\right]+\mu \lambda_{4}, \\
\lambda_{5}^{\prime}(t)= & \gamma_{0}\left[\lambda_{5}-\lambda_{3}\right]+\mu \lambda_{5},
\end{aligned}
$$

with transversality conditions

$$
\lambda_{1}\left(t_{f}\right)=\lambda_{2}\left(t_{f}\right)=\lambda_{3}\left(t_{f}\right)=\lambda_{4}\left(t_{f}\right)=\lambda_{5}\left(t_{f}\right)=0,
$$




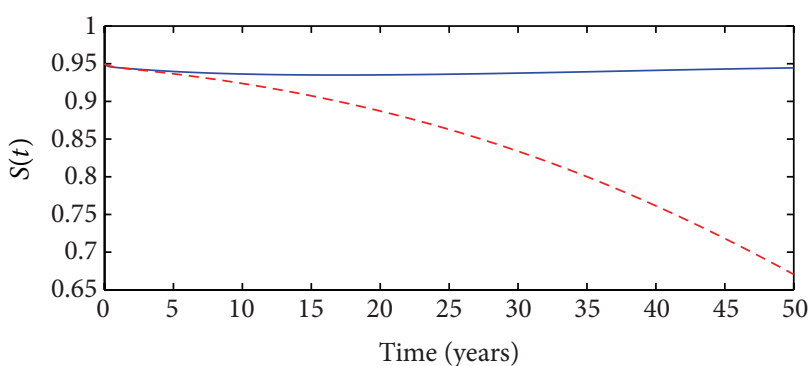

- With control

- - - Without control

(a)

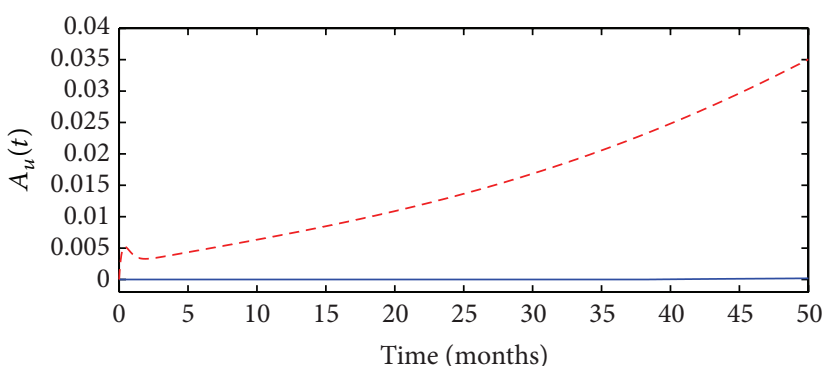

- With control - - - Without control

(c)

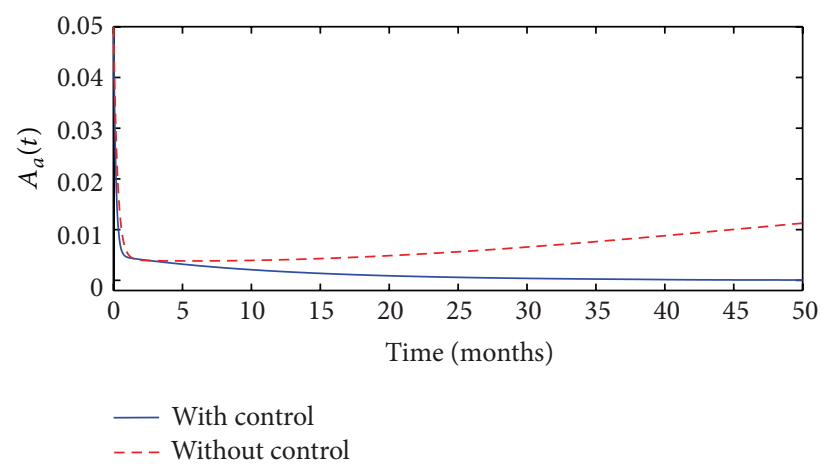

(b)

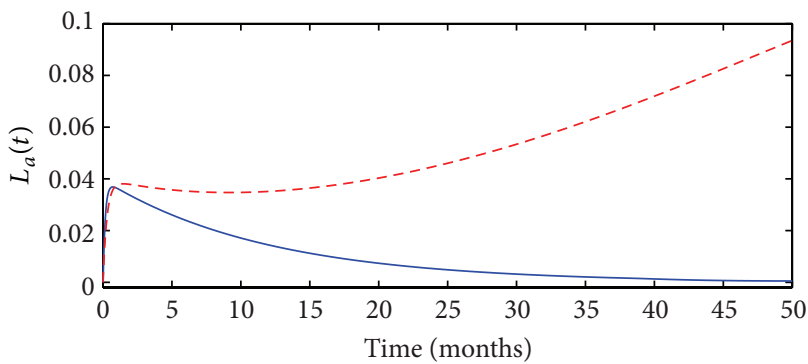

— With control

- - - Without control

(d)

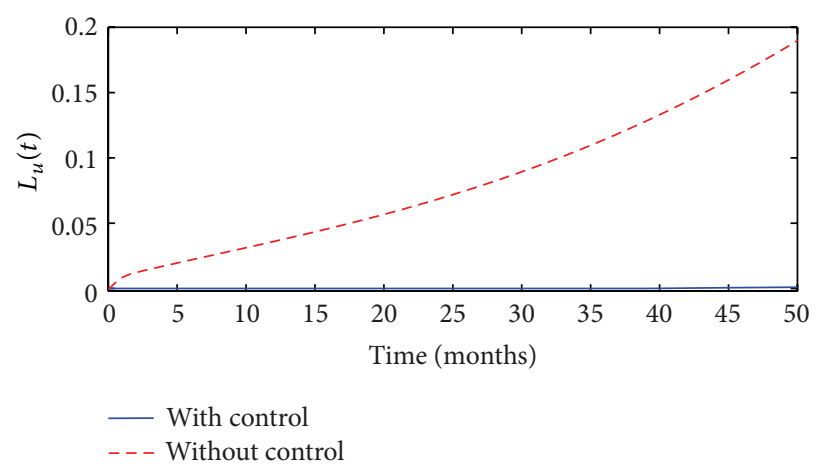

(e)

FIGURE 4: Graphs of the numerical solutions of the optimality system, showing propagation of (a) susceptibles $S$, (b) infected individuals with acute HSV-2 and also under antiviral treatment $A_{a}$, (c) infected individuals with latent HSV-2 after undergoing successful antiviral treatment $L_{a}$, (d) infected individuals with acute HSV-2 and not under antiviral treatment after quitting before completion $A_{u}$, and (e) the infected individuals with latent HSV-2 after undergoing natural healing $L_{u}$, over a period of 50 months.

and additionally the optimal control $u^{*}$ that minimizes $J(u)$ over $\mathcal{U}$ satisfies the optimality condition

$$
u^{*}=\max \left\{0, \min \left(1, \frac{\left(\lambda_{3}-\lambda_{2}\right) \delta A_{a}}{2 \vartheta}\right)\right\} \text {. }
$$

Proof. Corollary 4.1 of [37] gives the existence of an optimal control due to the convexity of the integrand of $J$ with respect to $u$, a priori boundedness of the state solutions, and the Lipschitz property of the state system with respect to the state variables. The differential equations governing the adjoint variables are obtained by differential of the Hamiltonian function, evaluated at the optimal control.
Due to the a priori boundedness of the state system, adjoint system, and the resulting Lipschitz structure of the ODEs, we obtain the uniqueness of the optimal control for small $t_{f}$. The uniqueness of the optimal control follows from the uniqueness of the optimality system, which consists of (54) and (55) with characterization (56). There is a restriction on the length of time interval in order to guarantee the uniqueness of the optimality system. The smallness restriction of the length of time is due to the opposite time orientations of (54) and (55), the state problem has initial values, and the adjoint variable has final values. The restriction is common in control problems [34, 35]. 
4.1. Numerical Simulations. The optimality system is solved using an iterative method with Runge-Kutta fourth-order scheme. Starting with a guess for the adjoint variables, the state equations are solved forward in time. Then these state values are used to solve the adjoint equations backward in time, and the iterations continue until convergence. The simulation was carried out using parameter values in Table 1 and the following values $z=0.95$ and $\vartheta=0.01$. The assumed initial conditions for the differential equations are $S=0.95$, $A_{a}=0.05, A_{u}=0.0, L_{a}=0.0$, and $L_{u}=0.0$.

In Figure 4(a) we note that for the whole interval under study optimal counselling and monitoring leads to a very small decrease of the susceptible population. In the absence of the control, it is worth noting that the population of the susceptible individuals drops drastically. Thus, optimal counselling and monitoring has an effect on the susceptible population.

Figure 4(b) illustrates the population of the individuals with acute HSV-2 and also under antiviral treatment in the presence and absence of the control over a period of 50 months. For both cases, in the presence and absence of the control there is not much difference since both populations have a sharp decrease for the period of $0-2.5$ months. For the period of 2.5-50 months, the population of the infected individuals in the absence of a control increases gradually. Further, we note that for the same period under review (2.550 months), the population of the individuals in the presence of the control reduces significantly signifying a positive effect of the control.

Figure 4(c) shows the individuals infected with acute HSV-2 and not under antiviral treatment after quitting before completion, in the presence and absence of the control. In the absence of the control, the individuals increase gradually in the first month and then reduce again a little, for the next two months before increasing rapidly for the remaining period under review, that is, 3-50 months. In the presence of the control, we note that for the whole period under review the HSV-2 cases remain very very low, relatively low, and relatively insignificant.

In Figure 4(d), the population of the infected individuals with latent HSV-2 after undergoing successful antiviral treatment in the presence and absence of the controls over a period of 50 months is presented. The population of the infected individuals with latent HSV-2 after undergoing successful antiviral treatment has a sharp increase for the first month, for both cases (with or without the control). In the presence of the control, we then note that from 2-50 months the population of the HSV-2 cases decreases gradually until it almost reaches 0 . In the absence of the control, the population of the individuals drops slightly over the period of 2-9 months; further, it then increases rapidly in the interval of 10-50 months.

In Figure 4(e) the dynamics of the infected individuals with latent HSV-2 after undergoing natural healing are presented. In the absence of the control, the population of the individuals increases rapidly for the whole period under review. In the absence of the control, the population of the infected individuals remains very very low for the whole period under review, that is, $0-50$ months.
Figure 5(a) represents the control $u$. The control is at the upper bound 1 for approximately 37 months and has a sharp drop until it reaches the lower bound 0 .

Figure 5(b) shows the control $u$, being varied. It shows that the weight constant has been varied (increased or reduced about $z=4$ ). Increasing $z$ implies increasing the minimization of $A_{u}$ and $A_{a}$ classes. Increasing the weight constant $z$ implies the control efforts on counselling and monitoring are implemented for a longer time. Further, reducing $z$ implies a reduction in the minimization of $A_{u}$ and $A_{u}$ classes. A reduction of the weight constant works against the efforts on counselling and monitoring since control will be implemented for a shorter period.

Figure 6 illustrates the range of values that can be taken by $\vartheta$ (increasing or decreasing it around the value 0.01 ). Increasing the weight and cost factor ( $\vartheta$ ) works against the effort on counseling and monitoring since it will be implemented for a shorter period. Reducing the weight and cost factor (9) works in favour of implementing the efforts on counselling and monitoring since it will be implemented for a longer period. It is worth noting that for our numerical simulations we assumed $\vartheta=0.01$, since that was the feasible optimum value which was applicable.

\section{Discussion}

HSV-2 is a significant factor for increased risk of acquisition and transmission of HIV and also being the leading disease in causing genital ulcers. In this study, a mathematical model to assess the impact of nonadherence to HSV-2 antiviral treatment is developed and analysed. Qualitative analysis of the model has shown that the model has a globally asymptotically stable disease-free equilibrium whenever the reproduction number is less than unity. The Lyapunov function approach was used to prove the global stability of the endemic equilibrium. Sensitivity analysis of the reproduction number suggested that increasing the rate of adherence by $51 \%$ has an effect of increasing the reproduction number by $51 \%$. Furthermore, analysis of the reproduction number through the contour plots shows that the reproduction number can be reduced to very low levels by increasing the adherence rate and decreasing the effective contact rate. Our results are in agreement with some earlier findings which managed to suggest that adherence to HSV-2 antiviral treatment is vital in the fight against HSV-2, although tailored adherence strategies may be needed $[38,39]$. Optimal control was then applied with the objective of minimizing the number of individuals quitting HSV-2 antiviral treatment. The technical tool used to determine the optimal strategy is the Pontryagin maximum principle. The control represents counselling and treatment. According to our optimal control results, we saw that a reduction of the weight constant works against the efforts on counselling and monitoring since they will be implemented for a shorter period. Our optimal control results also suggest that to successfully control HSV-2 prevalence we need to keep the cost of the control effort as low as possible. Overall, the study finds that in a community where HSV2 is endemic the disease can be eliminated or controlled if optimal intervention strategies are implemented. 


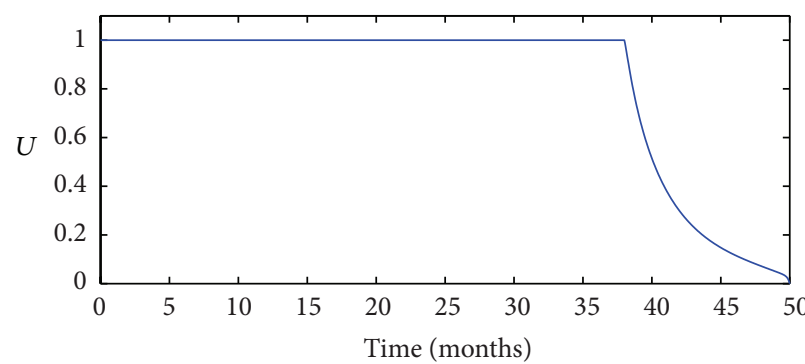

(a)

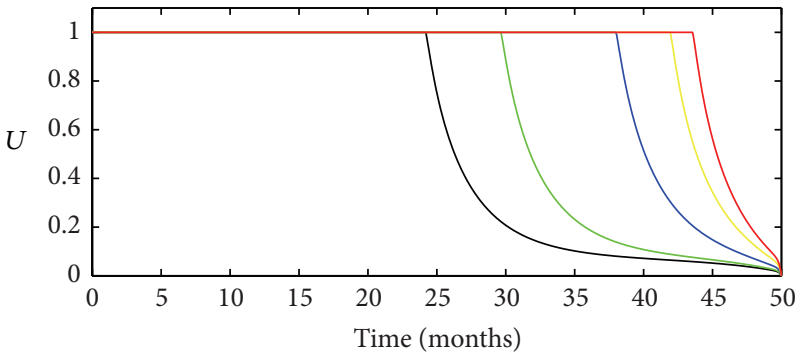

$\begin{aligned} z & =0.4 \\ z & =1.0 \\ z & =4.0\end{aligned}$

$z=8.0$

$z=11.0$

(b)

FIGURE 5: Graphs of the numerical solutions to the behaviour of the control constraint, (a) with the weight constant $z=0.4$ and (b) with the weight constant $z$ being varied.

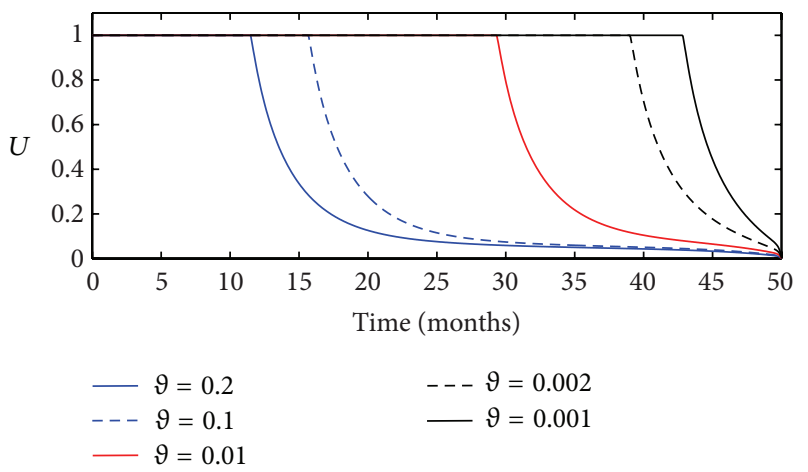

FIgURE 6: Graph of the numerical solutions to the behaviour of the control constraint, with the weight and cost factor $\vartheta$ being varied.

However just like any other model, we cannot say the model is complete; it can be extended to include resource limited or resource given communities.

\section{Conflict of Interests}

The authors declare that there is no conflict of interests regarding the publication of this paper.

\section{References}

[1] M. Laga, M. O. Diallo, and A. Buvé, "Interrelationship of sexually transmitted diseases and HIV: where are we now?" AIDS, vol. 8, no. 1, pp. S119-S124, 1994.

[2] S. O. Aral and J. N. Wasserheit, "STD-related health care seeking and health service delivery," in Sexually Transmitted Diseases, K. K. Holmes, P. F. Sparling, P. A. Mardh et al., Eds., pp. 1295-1306, McGraw-Hill, New York, NY, USA, 3rd edition, 1999.

[3] S. Kalichman and D. Rompa, "HIV treatment adherence and unprotected sex practices in people receiving antiretroviral therapy," Sexually Transmitted Infections, vol. 79, no. 1, pp. 59-61, 2003.

[4] S. Mushayabasa and C. P. Bhunu, "Modeling the impact of early therapy for latent tuberculosis patients and its optimal control analysis," The Journal of Biological Physics, vol. 39, no. 4, pp. 723747, 2013.

[5] A. J. Christensen, Patience Adherence to Medical Treatment Regimens: Bridging the Gap between Behavioral Science and Biomedicine, Yale University Press, New Haven, Conn, USA, 2004.

[6] P. J. McDonnell and M. R. Jacobs, "Hospital admissions resulting from preventable adverse drug reactions," Annals of Pharmacotherapy, vol. 36, no. 9, pp. 1331-1336, 2002.

[7] B. L. Senst, L. E. Achusim, R. P. Genest et al., "Practical approach to determining costs and frequency of adverse drug events in a health care network," American Journal of Health-System Pharmacy, vol. 58, no. 12, pp. 1126-1132, 2001.

[8] D. Watson-Jones, H. A. Weiss, M. Rusizoka et al., "Risk factors for herpes simplex virus type 2 and HIV among women at high risk in northwestern Tanzania: peparing for an HSV2 intervention trial," Journal of Acquired Immune Deficiency Syndromes, vol. 46, no. 5, pp. 631-642, 2007.

[9] Z. Feng, Z. Qiu, Z. Sang, C. Lorenzo, and J. Glasser, "Modeling the synergy between HSV-2 and HIV and potential impact of HSV-2 therapy," Mathematical Biosciences, vol. 245, no. 2, pp. 171-187, 2013.

[10] A. P. Fiddian, A. M. Halsos, B. R. Kinge, A. E. Nilsen, and K. Wikstrom, "Oral acyclovir in the treatment of genital herpes. Preliminary report of a multicenter trial," The American Journal of Medicine, vol. 73, no. 1, pp. 335-337, 1982.

[11] C. Celum, R. Levine, M. Weaver, and A. Wald, "Genital herpes and human immunodeficiency virus: double trouble," Bulletin of the World Health Organization, vol. 82, no. 6, pp. 447-453, 2004.

[12] H. B. Bosworth, Improving Patient Treatment Adherence. A Clinician's Guide, Springer, New York, NY, USA, 2010.

[13] M. L. Plummer, D. Watson-Jones, S. Lees et al., "A qualitative study of participant adherence in a randomized controlled trial of herpes suppressive therapy for HIV prevention in Tanzania," AIDS Care: Psychological and Socio-Medical Aspects of AIDS/HIV, vol. 22, no. 4, pp. 499-508, 2010.

[14] S. M. Blower, T. C. Porco, and G. Darby, "Predicting and preventing the emergence of antiviral drug resistance in HSV2," Nature Medicine, vol. 4, no. 6, pp. 673-678, 1998. 
[15] A. Mhlanga, C. P. Bhunu, and S. Mushayabasa, "HSV-2 and substance abuse amongst adolescents: insights through mathematical modelling," Journal of Applied Mathematics, vol. 2014, Article ID 104819, 17 pages, 2014.

[16] C. N. Podder and A. B. Gumel, "Transmission dynamics of a two-sex model for herpes simplex virus type-2," Canadian Applied Mathematics Quarterly, vol. 7, no. 2, pp. 339-386, 2009.

[17] S. Blower and P. Volberding, "What can modeling tell us about the threat of antiviral drug resistance?" Current Opinion in Infectious Diseases, vol. 15, no. 6, pp. 609-614, 2002.

[18] A. M. Foss, P. T. Vickerman, Z. Chalabi, P. Mayaud, M. Alary, and C. H. Watts, "Dynamic modeling of herpes simplex virus type-2 (HSV-2) transmission: issues in structural uncertainty," Bulletin of Mathematical Biology, vol. 71, no. 3, pp. 720-749, 2009.

[19] L. J. Abu-Raddad, A. S. Magaret, C. Celum et al., "Genital herpes has played a more important role than any other sexually transmitted infection in driving HIV prevalence in Africa," PLoS ONE, vol. 3, no. 5, Article ID e2230, 2008.

[20] D. R. Bangsberg, S. Perry, E. D. Charlebois et al., "Nonadherence to highly active antiretroviral therapy predicts progression to AIDS," AIDS, vol. 15, no. 9, pp. 1181-1183, 2001.

[21] C. E. Golin, H. Liu, R. D. Hays et al., "A prospective study of predictors of adherence to combination antiretroviral medication," Journal of General Internal Medicine, vol. 17, no. 10, pp. 756-765, 2002.

[22] P. Van den Driessche and J. Watmough, "Reproduction numbers and sub-threshold endemic equilibria for compartmental models of disease transmission," Mathematical Biosciences, vol. 180, no. 1-2, pp. 29-48, 2002.

[23] R. A. Horn and C. R. Johnson, Matrix Analysis, Cambridge University Press, Cambridge, UK, 1985.

[24] H. L. Smith and P. Waltman, The Theory of the Chemostat, Cambridge University Press, 1995.

[25] H. R. Thieme, "Persistence under relaxed point-dissipativity (with application to an endemic model)," SIAM Journal on Mathematical Analysis, vol. 24, no. 2, pp. 407-435, 1993.

[26] J. Li, Y. Yang, and Y. Zhou, "Global stability of an epidemic model with latent stage and vaccination," Nonlinear Analysis: Real World Applications, vol. 12, no. 4, pp. 2163-2173, 2011.

[27] H.-F. Huo and R. Chen, "Stability of an HIV/AIDS treatment model with different stages," Discrete Dynamics in Nature and Society, vol. 2015, Article ID 630503, 9 pages, 2015.

[28] D. R. Powell, J. Fair, R. J. Le Claire, L. M. Moore, and D. Thompson, "Sensitivity analysis of an infectious disease model," in Proceedings of the International System Dynamics Conference, Boston, Mass, USA, July 2005.

[29] L. M. Arriola and J. M. Hyman, "Being sensitive to uncertainty," Computing in Science and Engineering, vol. 9, no. 2, pp. 10-20, 2007.

[30] J. M. Wright, Y. Htun, M. G. Leong, P. Forman, and R. C. Ballard, "Evaluation of the use of calendar blister packaging on patient compliance with STD syndromic treatment regimens," Sexually Transmitted Diseases, vol. 26, no. 10, pp. 556-563, 1999.

[31] K. Beaucage, H. Lachance-Demers, T. T.-T. Ngo et al., "Telephone follow-up of patients receiving antibiotic prescriptions from community pharmacies," American Journal of HealthSystem Pharmacy, vol. 63, no. 6, pp. 557-563, 2006.

[32] H. Reyes, H. Guiscafre, O. Muñoz, R. Perez-Cuevas, H. Martinez, and G. Gutierrez, "Antibiotic noncompliance and waste in upper respiratory infections and acute diarrhea," Journal of Clinical Epidemiology, vol. 50, no. 11, pp. 1297-1304, 1997.
[33] P. Kardas, "Patient compliance with antibiotic treatment for respiratory tract infections," Journal of Antimicrobial Chemotherapy, vol. 49, no. 6, pp. 897-903, 2002.

[34] H. R. Joshi, "Optimal control of an HIV immunology model," Optimal control applications and methods, vol. 23, no. 4, pp. 199213, 2002.

[35] D. Kirschner, S. Lenhart, and S. Serbin, "Optimal control of the chemotherapy of HIV," Journal of Mathematical Biology, vol. 35, no. 7, pp. 775-792, 1997.

[36] L. S. Pontryagin, V. T. Boltyanskii, R. V. Gamkrelidze, and E. F. Mishchevko, The Mathematical Theory of Optimal Processes, vol. 4, Gordon and Breach Science Publishers, 1985.

[37] W. H. Fleming and R. W. Rishel, Deterministic and Stochastic Optimal Control, Springer, New York, NY, USA, 1975.

[38] M. Reitano, S. Tyring, W. Lang et al., "Valaciclovir for the suppression of recurrent genital herpes simplex virus infection: a large-scale dose range-finding study," Journal of Infectious Diseases, vol. 178, no. 3, pp. 603-610, 1998.

[39] D. Watson-Jones, K. Baisley, M. Rusizoka et al., "Measurement and predictors of adherence in a trial of HSV suppressive therapy in Tanzania," Contemporary Clinical Trials, vol. 30, no. 6, pp. 504-512, 2009. 


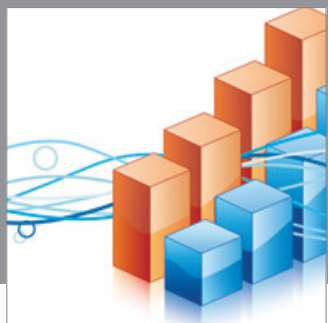

Advances in

Operations Research

mansans

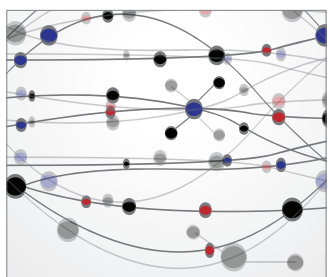

The Scientific World Journal
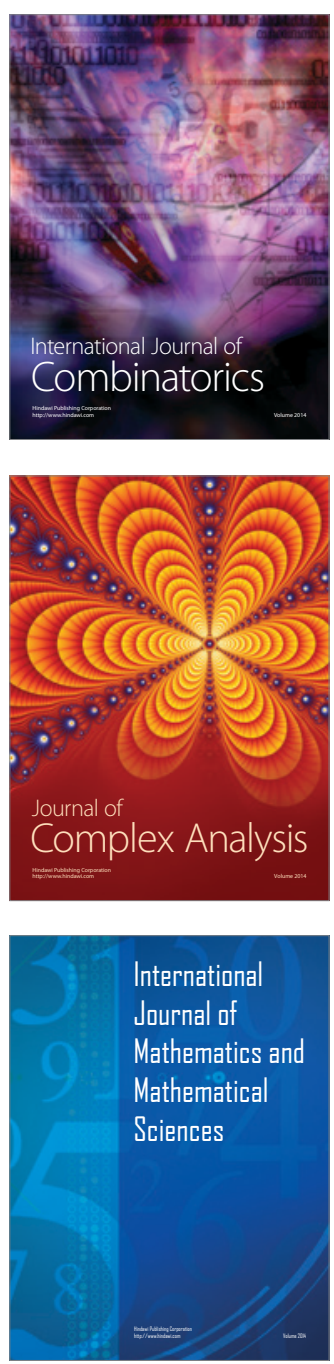
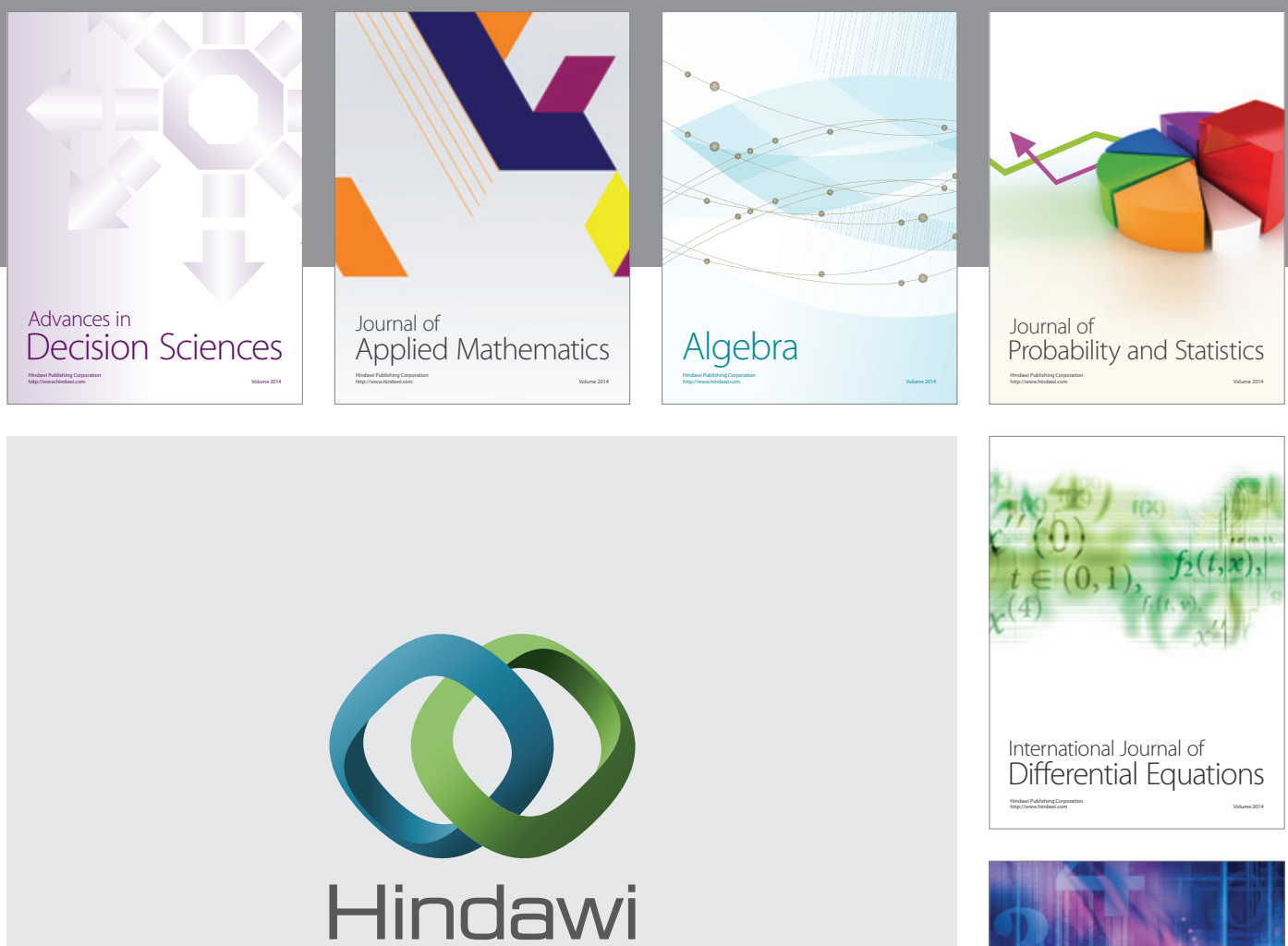

Submit your manuscripts at http://www.hindawi.com
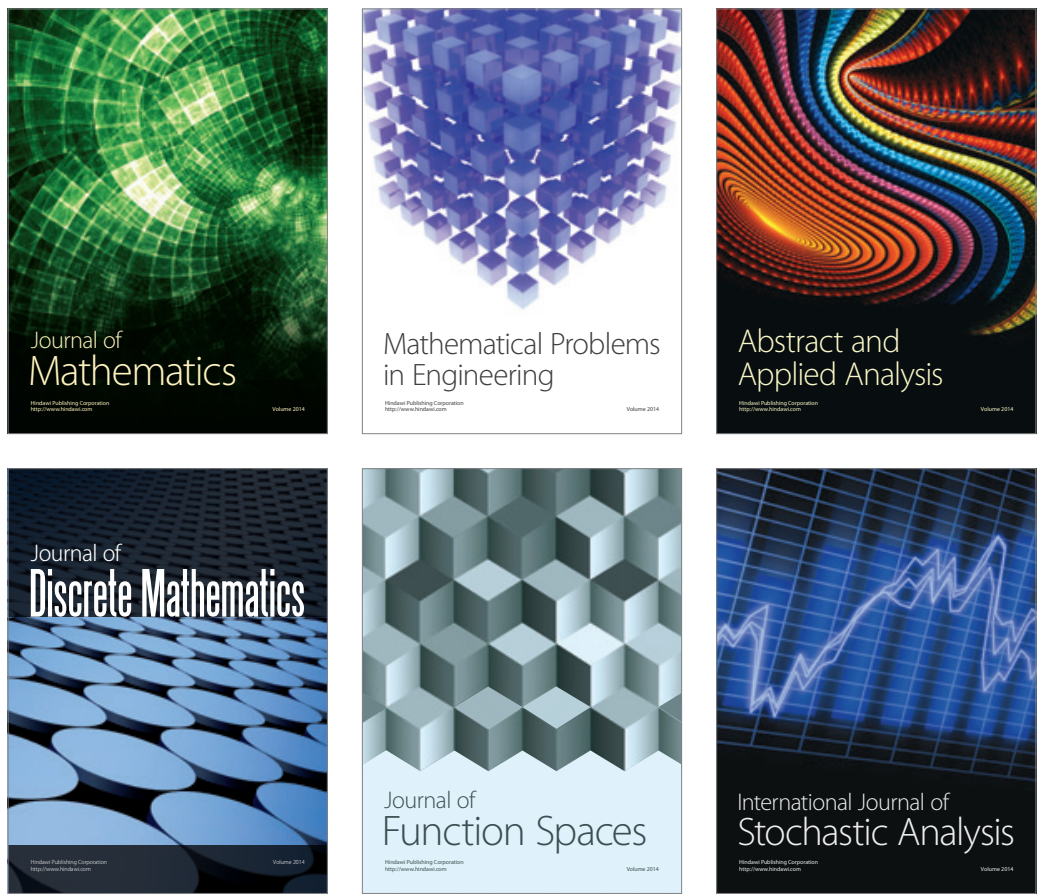

Journal of

Function Spaces

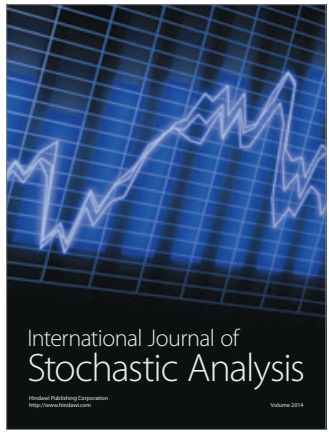

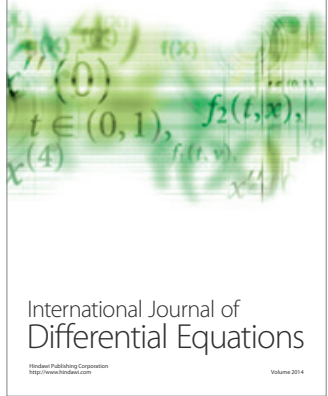
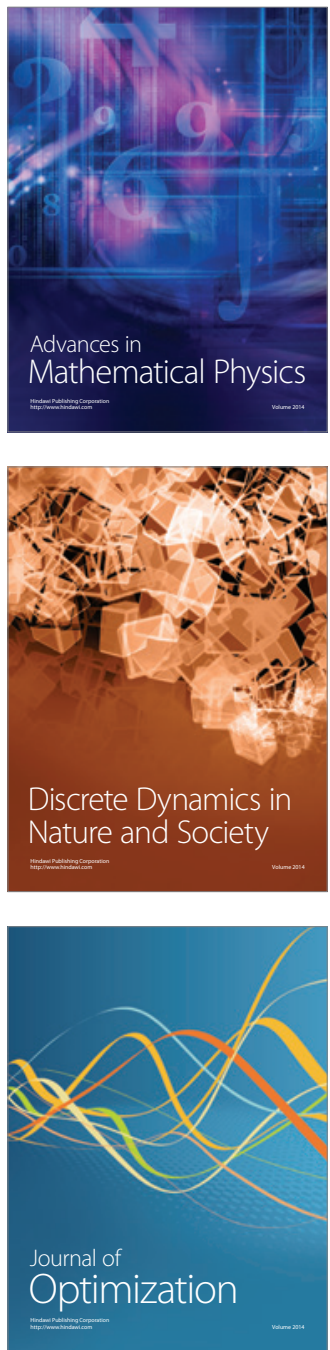